\title{
Drosophila have distinct activity-gated pathways that mediate attraction and aversion to $\mathrm{CO}_{2}$
}

\author{
Floris van Breugel*, Ainul Huda*, and Michael H. Dickinson* $\quad{ }^{*}$ California Institute of Technology, Pasadena CA 91125
}

Carbon dioxide is a volatile and broad signal of many organic processes, and serves as a convenient cue for insects in search of blood hosts ${ }^{1-6}$, flowers ${ }^{7}$, decaying matter ${ }^{8-11}$, communal nests ${ }^{12}$, fruit ${ }^{13}$, and wildfires ${ }^{14}$. Curiously, although Drosophila melanogaster feed on yeast that produce $\mathrm{CO}_{2}$ and ethanol during fermentation, laboratory experiments suggest that flies actively avoid $\mathrm{CO}_{2}{ }^{15-25}$. Here, we resolve this paradox by showing that both flying and walking fruit flies do actually find $\mathrm{CO}_{2}$ attractive, but only when they are in an active state associated with foraging. Aversion at low activity levels may be an adaptation to avoid $\mathrm{CO}_{2}$-seeking-parasites, or succumbing to respiratory acidosis in the presence of high concentrations of $\mathrm{CO}_{2}$ that are occasionally found in nature 26,27 . In contrast to $\mathrm{CO}_{2}$, flies are attracted to ethanol in all behavioral states, and invest twice as much time searching near ethanol compared to $\mathrm{CO}_{2}$. These behavioral differences reflect the fact that whereas $\mathrm{CO}_{2}$ is a generated by many natural processes, ethanol is a unique signature of yeast fermentation. Using genetic tools, we determined that the evolutionarily ancient ionotropic co-receptor IR25a is required for both $\mathrm{CO}_{2}$ and ethanol attraction, and that the receptors previously identified for $\mathrm{CO}_{2}$ avoidance are not involved. Our study lays the foundation for future research to determine the neural circuits underlying both state- and odorant- dependent decision making in Drosophila.

The life history of the fruit fly, Drosophila melanogaster, revolves around fermenting fruits, where they feed, mate, and deposit eggs. Their lifecycle from egg to adult takes approximately 10-14 days, roughly the same amount of time that most ripe fruit takes to decay. Thus, upon emerging from their puparia, adult flies need to locate a fresh ferment. The primary compounds produced by yeast fermentation are ethanol and $\mathrm{CO}_{2}$. Because of its high volatility, $\mathrm{CO}_{2}$ emission is greatest near the start of fermentation, whereas the ethanol emission increases more slowly (Fig. 1a). Other odors associated with fermentation, such as acetic acid and ethyl acetate, form later when bacteria begin to break down the ethanol.

To find an active rot, flies should therefore search near sources of both $\mathrm{CO}_{2}$ and ethanol. Variable air currents make it difficult to estimate the exact concentration of $\mathrm{CO}_{2}$ emitted from ferments in the wild; however, we measured the $\mathrm{CO}_{2}$ concentration in $500 \mathrm{~mL}$ bottles used to rear flies in many laboratories (see Methods and Fig. S1). Such bottles contain $0.5-1 \% \mathrm{CO}_{2}$ depending on the amount of yeast and flies present (Fig. 1b), and serve as effective traps if left without a lid. In trap assays (Fig. 1c), Drosophila showed a preference for 2-day-old apple juice ferments compared to older solutions in which the yeast had flocculated and were no longer producing $\mathrm{CO}_{2}$ (Fig. 1d).

This casual evidence that $\mathrm{CO}_{2}$ attracts Drosophila contradicts many prior studies that concluded flies actively avoid $\mathrm{CO}_{2}$ in small chambers and T-mazes ${ }^{15-25}$. To study how flies respond to different odors under more ethologically relevant conditions, we recorded the flight trajectories ${ }^{28,29}$ of flies in a wind tunnel containing a fruit-sized landing platform, which we programmed to periodically release plumes of $\mathrm{CO}_{2}$ or ethanol (Fig. 2a-b). In the presence of either
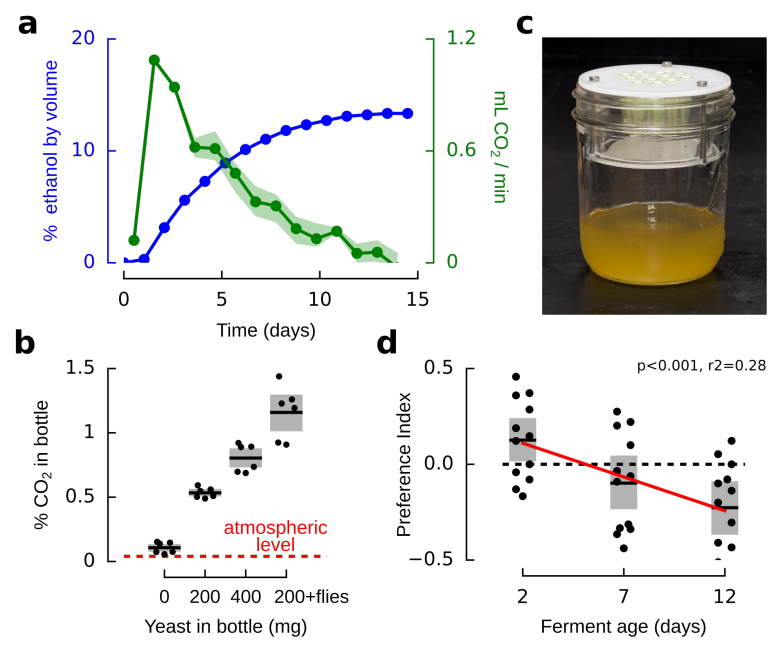

Figure $1 \mid$ Drosophila prefer early fermentations, at peak $\mathrm{CO}_{2}$ production. a, Alcohol by volume for apple juice and sugar fermented with champagne yeast over the course of 2 weeks, measured with a hydrometer. $\mathrm{CO}_{2}$ production was calculated from the stoichiometry of fermentation (1 sugar molecule yields 2 ethanol $+2 \mathrm{CO}_{2}$ ), corresponding to the derivative of alcohol by volume. b, $\mathrm{CO}_{2}$ concentration in $500 \mathrm{~mL}$ fly rearing bottles under common laboratory conditions. See Fig. S1 for methods and calibration details. c, Trap assay. d, Preference index exhibited by flies in three 2 -choice assays, using traps shown in $\mathbf{b}$. Flies were presented with two traps: one was a completed 14 day old ferment which had been stored in the refrigerator, the second was a fresh ferment aged 2, 7, or 12 days old. Positive preference index indicates a preference for the fresh ferment. Mean and standard deviation of total captured flies for each trial: 105 \pm 59 . In all panels, shading indicates bootstrapped $95 \%$ confidence intervals. 
odor, flies were far more likely to approach and land on the platform. They also approached a dark spot on the floor of the wind tunnel (Fig. 2c-d), consistent with prior experiments with flies and mosquitoes ${ }^{2,29}$. Flies were more likely to approach the platform or the dark spot in the presence of ethanol compared to $\mathrm{CO}_{2}$, but were equally likely to land in the presence of either odor (Fig. 2e).

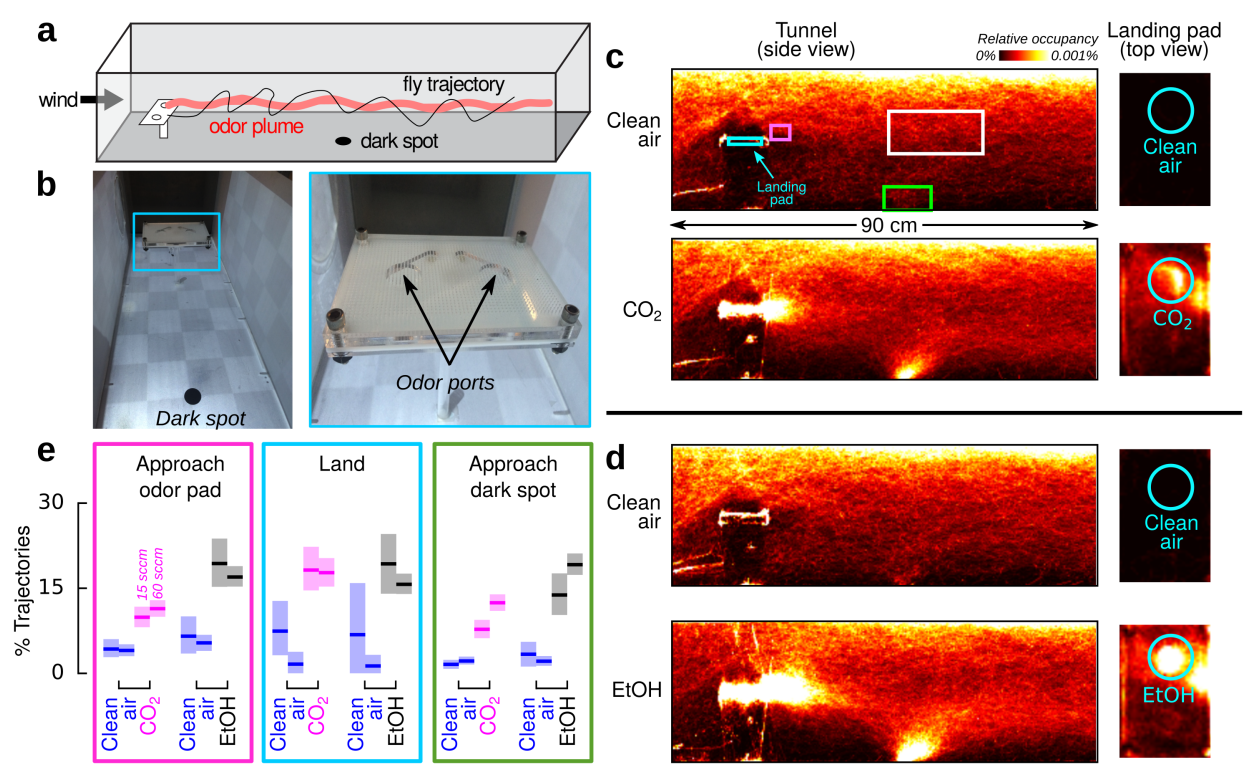

Figure $2 \mid$ Drosophila are attracted to both ethanol and $\mathrm{CO}_{2}$ in flight. a, Diagram of our wind tunnel, illustrating the relative position of the odor platform and a conspicuous visual feature. $\mathbf{b}$, Photograph of the wind tunnel, viewing upwind, and close up of the odor-emitting landing platform. $\mathbf{c}$, $\mathbf{d}$ Heat-maps indicating relative occupancy of flies in the presence of either $\mathrm{CO}_{2}$ or ethanol. Cohorts of 12 flies were introduced into the wind tunnel and their behavior recorded over the course of $16 \mathrm{hrs}$. Throughout the experiment, $100 \mathrm{sccm}$ of clean air emerged from both odor ports. For 30 min every hour, $60 \mathrm{sccm}$ of either $\mathrm{CO}_{2}$ or clean air bubbled through pure ethanol was added to one of the odor ports. Control data come from the $30 \mathrm{~min}$ segments of clean air prior to each odor stimulus. Number of cohorts: $9\left(\mathrm{CO}_{2}\right), 6$ (ethanol). Number of trajectories total: 59,900 - 101,000 per panel. e, Fraction of trajectories from $\mathbf{c}$ and $\mathbf{d}$ that enter one of the colored volumes in $\mathbf{c}$ relative to another volume. Approaches to landing pad: magenta/white; landings: cyan/magenta; approaches to dark spot: green/white. Number of trajectories per condition: 44-1288 (control), 228-1815 (odor). Experiments were performed on two different flowrates (i.e. concentrations): $15 \mathrm{sccm}$ and $60 \mathrm{sccm}$. In all panels, shading indicates bootstrapped $95 \%$ confidence intervals.

To quantify the behavior of flies after they land, we designed a new platform suitable for automated tracking (Fig. 3a-b). For a flow rate of $60 \mathrm{sccm} \mathrm{CO}$, the $\mathrm{CO}_{2}$ concentration near the surface of the platform was approximately 3\% (Fig. 3c, S2). After landing near a source of $\mathrm{CO}_{2}$, ethanol, or apple cider vinegar, flies exhibited local search behavior (Fig. 3d), which we summarized using four descriptive statistics (Fig. 3e, S3). Flies spent approximately twice as much time exploring the platform in the presence of ethanol compared to $\mathrm{CO}_{2}$ or any other odor. Vinegar elicited smaller local searches than either $\mathrm{CO}_{2}$ or ethanol. While searching on the platform, flies approached the odor source most frequently for ethanol and $\mathrm{CO}_{2}$. Vinegar elicited slightly fewer approaches compared to $\mathrm{CO}_{2}$, consistent with the hypothesis that vinegar might indicate a less favorable, late-stage ferment. Flies spent significantly less time standing still on the platform in the presence of $\mathrm{CO}_{2}$ compared to any other odor, exhibiting an overall mean walking speed greater than $2 \mathrm{~mm} \mathrm{~s}^{-1}$. When combined in a single odor stream, $\mathrm{CO}_{2}$ and ethanol together elicited a stronger search behavior than that exhibited to either odor alone.

One prior study using a tethered flight assay showed that Drosophila are attracted to $\mathrm{CO}_{2}$ while flying, a result that was attributed to the influence of the elevated levels of octopamine during flight ${ }^{30}$. Our results confirm this observation in freelyflying flies; however, we also found that flies continue to be attracted to $\mathrm{CO}_{2}$ after they land. One possible explanation for this discrepancy is that the elevated levels of octopamine during flight might influence the flies' reactions to $\mathrm{CO}_{2}$ for a short time after landing. To test this hypothesis, we built an enclosed walking arena in which flies were unable to fly (Fig. 4a, S4-6), and presented them with pulses of $5 \% \mathrm{CO}_{2}$ (close to the 3\% concentration that elicited attraction in the wind tunnel assay). Starved flies presented with $\mathrm{CO}_{2}$ after acclimating to the arena for $10 \mathrm{~min}$ exhibited aversion, as has been previously reported in such chambers (Fig. 4b). However, if allowed to acclimate for two hours and then given a pulse of $\mathrm{CO}_{2}$, the animals exhibited attraction (Fig. 4c). 
To study their responses to $\mathrm{CO}_{2}$ in more detail, we recorded the behavior of flies for 20 continuous hours in darkness, while offering $10 \mathrm{~min}$ long presentations of $\mathrm{CO}_{2}$ from alternating sides of the arena every 40 minutes (Fig. 4d). Throughout the experiments, both sides of the arena received $20 \mathrm{sccm}$ of air saturated with water vapor. The flies exhibited a clear circadian rhythm in their activity within the chamber, as indicated by their mean walking speed. At times of peak activity - near their entrained dusk and dawn — flies showed a strong initial attraction to $\mathrm{CO}_{2}$, which decayed stereotypically during the 10 min presentation. At times of low activity - at mid-day and during the night — the flies exhibited a mild aversion to $\mathrm{CO}_{2}$. Starving flies for 24 hours prior to placing them in the chamber (instead of just 3 hours) changed their activity profile, resulting in a slightly elevated attraction during their subjective night. Ethanol, in contrast, elicited sustained attraction regardless of baseline activity or time of day (Fig. 4d).
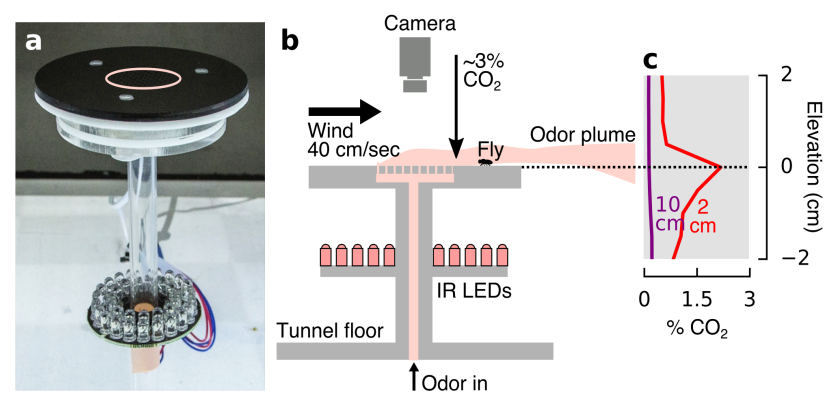

d
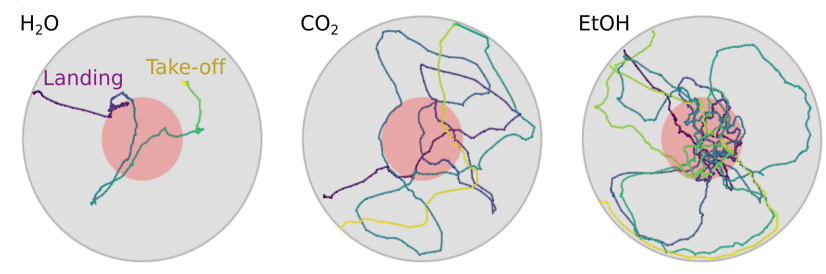

e
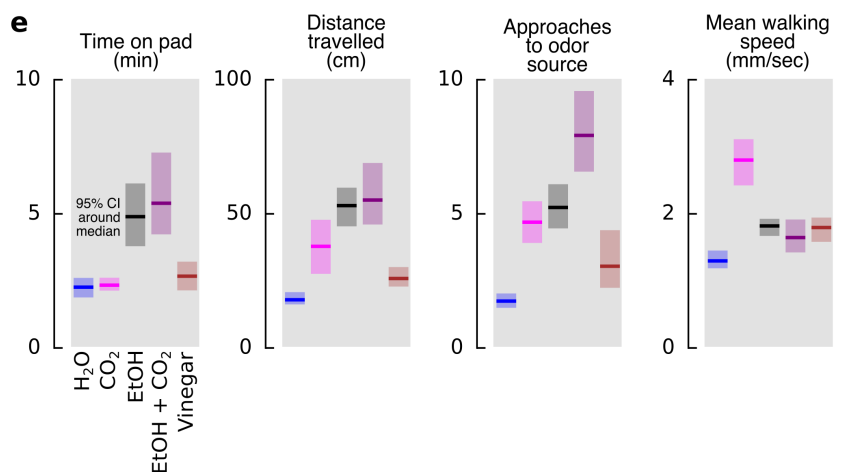

Figure 3 | Drosophila are attracted to $\mathrm{CO}_{2}$ while walking, but spends twice as much time exploring ethanol sources. a, Photograph of landing platform for observing walking behavior. $\mathbf{b}$, Cross-sectional diagram of the odor platform. $\mathbf{c}, \mathrm{CO}_{2}$ concentration profile for two altitude transects $2 \mathrm{~cm}$ and $10 \mathrm{~cm}$ downwind from the platform at a $60 \mathrm{sccm}$ flow rate (see supplemental materials, Fig. S2). d, Stereotypical trajectories in response to odors. Color encodes time. e, Four descriptive statistics summarizing flies' behavior on the platform in response to different odors. We used a flow rate of 60 sccm for each odor, except for the ethanol $+\mathrm{CO}_{2}$ combination which consisted of $60 \mathrm{sccm}$ clean air bubbled through ethanol with $15 \mathrm{sccm}$ of $\mathrm{CO}_{2}$ added. See Fig. $\mathrm{S} 3$ for experiments done at additional flow rates. Horizontal bars: median value; shading: 95\% confidence interval. Number of trajectories for each case is between 121 and 193.
Our experiments thus far suggest a possible correlation between activity and attraction to $\mathrm{CO}_{2}$. To test this hypothesis, we made several other environmental manipulations that are known to alter activity: increased temperature and wind speed (Fig. 4e). When we increased our bulk flow rate to $100 \mathrm{sccm}$, flies exhibited a peak walking speed (at dusk) of about $1.5 \mathrm{~mm} \mathrm{~s}^{-1}$, nearly half the speed we measured when using a flow rate of 20 sccm. This result is consistent with observations that flies stop moving in the presence of wind ${ }^{31}$. Instead of showing attraction, these flies exhibited aversion to $5 \% \mathrm{CO}_{2}$ when it was presented at this higher flow rate; however, they still exhibited attraction to ethanol (Fig. 4e). This result helps to explain why previous studies that used high bulk airflow rates of $100-1000 \mathrm{sccm}$ to present $\mathrm{CO}_{2}{ }^{16,24}$ observed aversion. To further explore the effect of wind speed on behavior, we clipped the flies' aristae. This manipulation destroys their primary means of detecting airflow but does not interfere with the detection of odors ${ }^{32}$. The aristaeless flies exhibited the same walking speed and attraction to $\mathrm{CO}_{2}$ at the high flow rate as exhibited by normal flies at the low flow rate. We also warmed flies with intact aristae to $32^{\circ} \mathrm{C}$, which increased their baseline activity. These flies also exhibited attraction to $\mathrm{CO}_{2}$ at the higher flow rate. Pooling data across all our experimental conditions, we found that flies were attracted to $\mathrm{CO}_{2}$ when they had a baseline walking speed above $\sim 2.4 \mathrm{~mm} \mathrm{~s}^{-1}$ (Fig. 4f). This result is similar to the mean walking speed value we observed in our wind tunnel assay, which was higher for $\mathrm{CO}_{2}$ than the other odors we tested. This suggests that there may be some underlying physiological connection between circuits regulating locomotor activity and those regulating $\mathrm{CO}_{2}$ attraction. To confirm that activity dependent attraction to $\mathrm{CO}_{2}$ is not a function of social interactions, we also performed experiments on 30 single flies, which on average behaved exactly as the cohorts of 10 (Fig. S7). We also tested three concentrations of $\mathrm{CO}_{2}(1.7 \%, 5 \%, 15 \%)$ and found that $5 \%$ elicited the strongest response, consistent with our wind tunnel experiments (Fig. S8).

Although flies' responses to ethanol and $\mathrm{CO}_{2}$ were similar during the first minute of the stimulus, the attraction to ethanol was more sustained. The time course of behavior was remarkably similar in the walking arena and wind tunnel (Fig. 4g-j), suggesting that the behavioral dynamics of olfactory attraction are robust to the stimulus environment, and may represent an adaptation for utilizing information that ecologically broad $\left(\mathrm{CO}_{2}\right)$ and more specific (ethanol) odorants provide. 


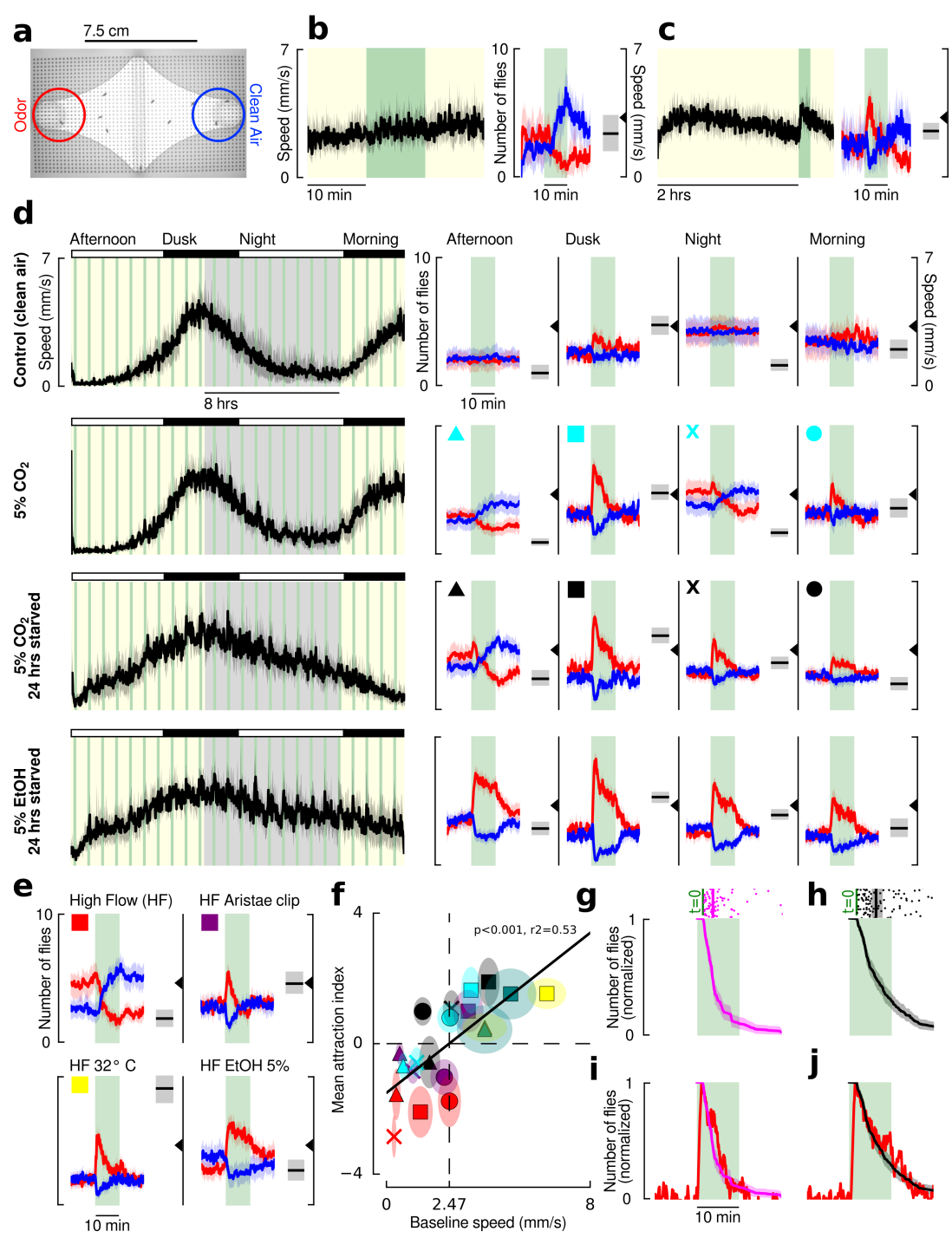

Figure 4 | Drosophila are briefly attracted to $\mathrm{CO}_{2}$ during periods of high activity, in contrast to their activity independent and sustained attraction to ethanol. a, Image of walking arena, with blue and red regions of interest (ROI) for counting flies near clean air and odor.

b, (left) Mean speed of 10 starved flies over $30 \mathrm{~min}$. Green indicates times when $1 \mathrm{sccm}$ of odor (or clean air control) was added to the constant humidified clean air bulk flow $\left(20 \mathrm{sccm}\right.$ ) from alternating sides. (right) Number of flies near the $\mathrm{CO}_{2}$ (red) and clean air (blue) over time. Black bar and shading shows the mean and $95 \%$ confidence interval of the flies' speeds five min prior to the odor presentation. The triangle provides a reference point. $\mathrm{N}=8$ cohorts of 10 flies. $\mathbf{c}$, Same as $\mathbf{b}$, but with a 2-hour acclimatization period prior to the $\mathrm{CO}_{2}$ presentation. $\mathrm{N}=10$ cohorts of 10 flies each. Experiments for $\mathbf{b}$ and $\mathbf{c}$ were both done during flies' peak activity time (dusk). d, (left) Mean speed of flies for a 20 hr experiment. Yellow/gray indicate entrained day/night cycle (experiments are done in darkness). (right) Data plotted as in $\mathbf{b}$, for four different time frames. e, Data for the dusk time frame of experiments done under $100 \mathrm{sccm}$ bulk flow conditions. In these experiments $5 \mathrm{sccm}$ odor was added to achieve the same concentration as in e. Experiments were performed with $5 \% \mathrm{CO}_{2}$ stimuli on in-tact flies (red), flies with aristae surgically removed (purple), and in-tact flies at $32^{\circ}$ under a heat lamp (yellow). Finally, we performed experiments with in-tact flies in response to ethanol. $\mathbf{f}$, Summary of $\mathrm{CO}_{2}$ responses presented in $\mathbf{d}$ and $\mathbf{e}$ (color and shape encodes experiment and time of day). Green data points are for experiments done at $20 \mathrm{sccm}$ bulk flow at $32^{\circ} \mathrm{C}$. Mean attraction index is calculated as the mean number of flies near the $\mathrm{CO}_{2}$ over the 10 - min presentation, minus the number of flies near the $\mathrm{CO}_{2} 5$ min prior to the presentation. Baseline speed refers to the mean speed of all the flies 5 min prior to the $\mathrm{CO}_{2}$ presentation. $\mathrm{g}$, Scattergram shows the amount of time each fly spent searching the odor platform in the wind tunnel from Fig. $3 \mathrm{a}$ in the presence of $60 \mathrm{sccm}$ CO2 (data is repeated from Fig. 3e). Time trace is the bootstrapped mean and $95 \%$ confidence intervals for the normalized number of flies that would have been on the platform had all the flies landed simultaneously. The green shading is only provided for reference - the odor never turned off in these wind tunnel experiments. $\mathbf{h}$, Same as $\mathrm{g}$, but for ethanol. $\mathbf{i}$, Time trace from $\mathbf{g}$ overlaid on the normalized number of un-starved flies near the $5 \%$ CO2 source during the dusk time period in the walking arena, copied from panel d. $\mathbf{j}$, Time trace from $\mathbf{h}$ overlaid on the normalized number of un-starved flies near the $5 \%$ ethanol source during the dusk time period in the walking arena, data not shown in $\mathbf{d}$. We chose un-starved flies for the comparisons because wind tunnel experiments were done with un-starved flies. We chose the $60 \mathrm{sccm}$ case for the comparison because the $\mathrm{CO}_{2}$ concentration in the wind tunnel matches the $5 \% \mathrm{CO} 2$ stimulus given in the walking experiments. Throughout the figure, shading around data indicates $95 \%$ confidence intervals. All experimental combinations were performed with 6 cohorts of 10 flies each.

Despite the ethological importance of both ethanol and $\mathrm{CO}_{2}$ as food cues for Drosophila, the olfactory receptors used to detect these odors during foraging are not known. To determine if $\mathrm{CO}_{2}$ attraction is mediated by either an olfactory (OR) or 
ionotropic (IR) receptor, we used our apparatus to test an IR8a; IR25a; Orco, Gr63a quadruple mutant, which lack the OR and IR co-receptors as well as a $\mathrm{CO}_{2}$-sensitive gustatory receptor (Fig. 5a-b). These near-anosmic mutants exhibited no detectable behavioral response to $\mathrm{CO}_{2}$. Flies in which we surgically removed the $3^{\text {rd }}$ antennal segment also showed no response to $\mathrm{CO}_{2}$, despite otherwise normal levels of activity. Together with our arista ablations (Fig. 4e), these experiments show that $\mathrm{CO}_{2}$ attraction is mediated by the olfactory system in the $3^{\text {rd }}$ antennal segment.

Prior research has shown that flies' aversion to $\mathrm{CO}_{2}$ is mediated by a pair of olfactory receptors, Gr63a and Gr21 $\mathrm{a}^{15,18,33}$, with high concentrations of $\mathrm{CO}_{2}$ also being detected by the acid-sensitive ionotropic receptor IR64a ${ }^{20}$ (which operates together with the co-receptor IR8a). Mutant flies lacking the IR64a receptor showed no significant change in their behavior compared to wild type (Fig. 5c). Mutants lacking the Gr63a receptor exhibited no aversion to $\mathrm{CO}_{2}$ (Fig. 5c), consistent with the prior literature; however, the same animals were still attracted to $\mathrm{CO}_{2}$ when more active. Homozygous Gr63a/IR64a double mutants behaved similarly to the Gr63a mutants. It is noteworthy that the characteristic decaying time course of attraction was unaffected in Gr63a mutants, even though these flies showed no aversion. This suggests that the decay in attraction to $\mathrm{CO}_{2}$ is not caused by an increase in aversion over time.

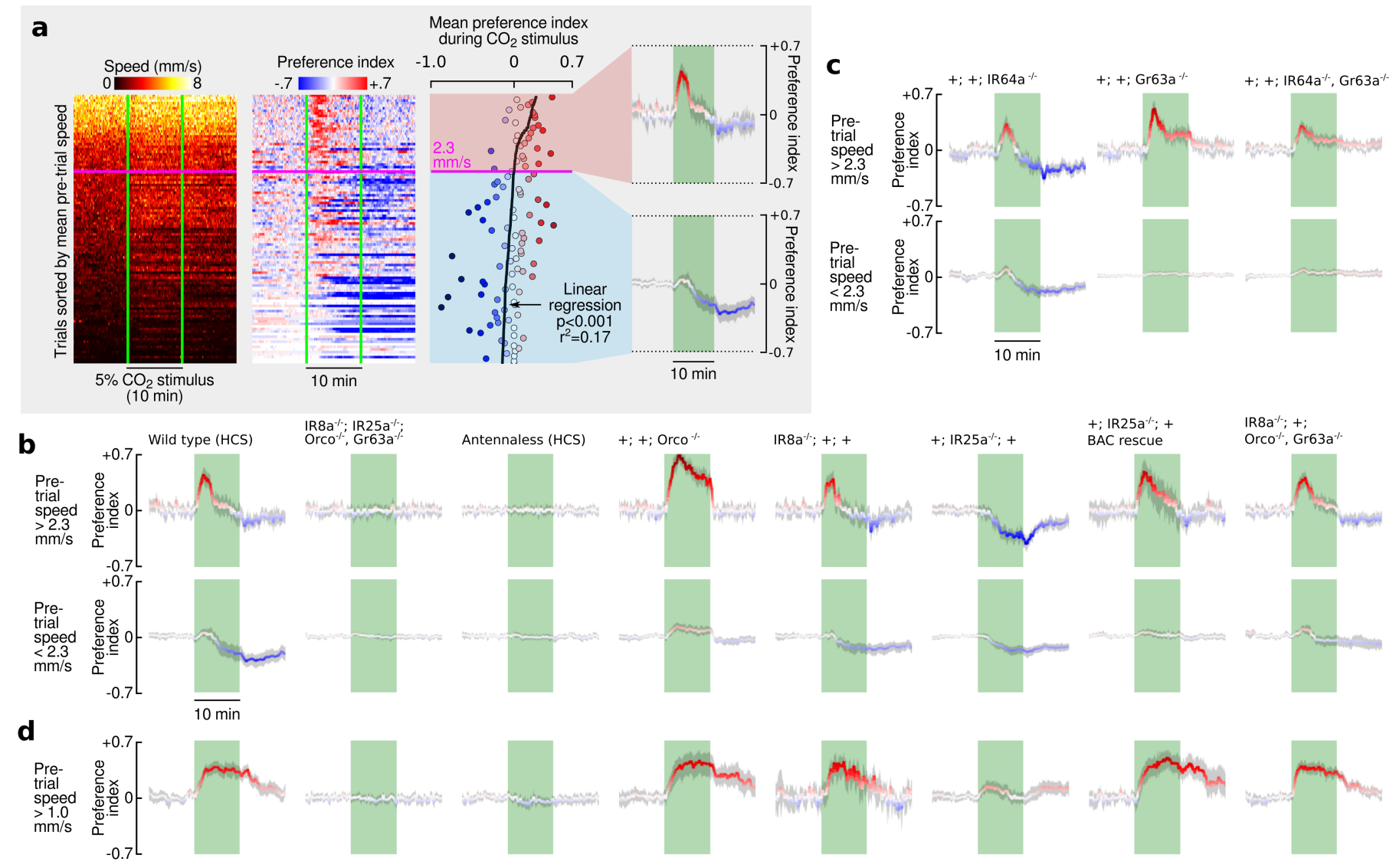

Figure 5 | $\mathbf{C O}_{2}$ and ethanol attraction in Drosophila requires IR25a, but not Orco, IR8a, or Gr63a. a, Data from 10 cohorts of flies with a 5\% $\mathrm{CO}_{2}$ stimulus sorted by the mean speed (S) during the reference period of 5 min prior to the odor stimulus $\left(\left.\bar{S}\right|_{\text {Ref }}\right)$. To achieve a wide range of baseline activities, 4 of the cohorts were starved for 24 hrs, 3 were starved for $3 \mathrm{hrs}$, and 3 were starved for 3 hrs and heated to $32^{\circ} \mathrm{C}$. Preference index (PI) was calculated in two steps: (1) $\mathrm{PI}_{0}=\left(\mathrm{N}_{\text {odor }}-\mathrm{N}_{\text {control }}\right) / \mathrm{N}_{\text {total }}$; (2) $P I=P I_{0}-\left.\overline{P I_{0}}\right|_{\text {Ref }}$. Where $\mathrm{N}$ denotes the number of flies, and $\mathrm{N}_{\text {total }}=10$. Next, we calculated the mean PI during the stimulus period and determined the linear regression with respect to $\left.\bar{S}\right|_{\text {Ref }}$, and used the intercept to cluster the data into two groups: high activity and low activity. For these two groups, we calculated the mean PI over time. b-c, Data plotted as in last panel of a, for different manipulations and mutants, using the intercept found in a of $2.3 \mathrm{~mm} / \mathrm{sec}$ to cluster the data. All flies were presented with randomly interleaved stimuli of $0 \%$ or $5 \%$; only responses to $5 \%$ are shown here. Responses to $0 \%$ stimuli are shown in Fig. S9. IR 25 a BAC flies did not respond to $\mathrm{CO}_{2}$ when heated; only the unheated data is shown here (see Fig. S10). d, Same as b, but for ethanol odors. Since ethanol attraction is not activity dependent, we used 5 cohorts of $24 \mathrm{hr}$ starved flies, and used a mean speed of $1 \mathrm{~mm} / \mathrm{sec}$ to cluster the data. Only the higher activity is shown; the low activity flies show small responses ethanol. Shading indicates bootstrapped $95 \%$ confidence intervals for all panels.

Collectively, our results suggest that none of the canonical $\mathrm{CO}_{2}$ receptors are responsible for attraction. The OR class of receptors is unlikely to mediate the response; indeed, Orco mutants exhibited a sustained attraction to $\mathrm{CO}_{2}$. IR8a mutants also 
exhibit normal attraction to $\mathrm{CO}_{2}$. Ir25a mutants, however, exhibited only aversion to $\mathrm{CO}_{2}$ at all activity levels, whereas rescuing Ir25a with a bacterial artificial chromosome ${ }^{34}$ rescued their attraction. Mutant flies lacking Orco, Ir8a, and Gr63a exhibit wild type attraction to $\mathrm{CO}_{2}$, indicating that none of these proteins are necessary for $\mathrm{CO}_{2}$ attraction. We further verified our results by testing a different mutant allele of the IR25a, which behaved in the same manner (Fig. S10).

Although ethanol is the most ethologically relevant cue related to fermentation and elicits the strongest attraction, the receptors for ethanol are still not known. We used the same set of co-receptor mutants to determine that IR25a, but not Orco or IR8a, is required for attraction to ethanol (Fig. 5d). IR25a is, however, not required for attraction to other odors associated with foraging, like apple cider vinegar (Fig. S9), confirming that these mutants are still capable of exhibiting attractive behaviors.

Prior studies reporting aversion to $\mathrm{CO}_{2}$ have suggested that it serves as a pheromonal cue (Drosophila Stress Odor, DSO) by which stressed flies signal others to flee a local enviroment ${ }^{15}$. Our result that active flies are attracted to $\mathrm{CO}_{2}$ is not consistent with this hypothesis. An alternative explanation for the prior findings is that stressed insects release $\mathrm{CO}_{2}$ simply because they have it stored in their tracheal system as part of the normal process of discontinuous respiration ${ }^{35,36}$. Indeed, we found that even mosquitoes (which are strongly attracted to $\mathrm{CO}_{2}$ ) release $\mathrm{CO}_{2}$ when shaken (Fig. S12). We suggest that the DSO hypothesis is a by-product of two unrelated behaviors: the release of tracheal $\mathrm{CO}_{2}$ by agitated flies and the avoidance of $\mathrm{CO}_{2}$ while in a behavioral state related to either low activity levels or being recently introduced to a new chamber (and thus likely to be in a behavioral state more associated with exploring a new environment than foraging). This aversive behavior may be an adaptation that helps sleeping flies either minimize encounters with parasites that are themselves attracted to $\mathrm{CO}_{2}$ as a means of finding hosts (parasitic wasps of Drosopbila are attracted to yeast products ${ }^{37}$ and thus likely $\mathrm{CO}_{2}$; other hematophagous parasites are often attracted to $\mathrm{CO}_{2}^{1,3,4,6}$ ), or avoid succumbing to respiratory acidosis in the presence of high concentrations of $\mathrm{CO}_{2}$. Examples of insects being fatally attracted to high levels of $\mathrm{CO}_{2}$ have been reported in the literature ${ }^{27}$, and we have replicated this behavior in the lab (Fig. S13).

Our study adds Drosophila to the long list of insects that are attracted to $\mathrm{CO}_{2}{ }^{38}$. This implies an ancient evolutionary role of $\mathrm{CO}_{2}$ in insect behavior, as well as a highly conserved means for detecting it. These hypotheses are supported by our finding that $\mathrm{CO}_{2}$ attraction in Drosophila requires the ionotropic co-receptor IR25a, the most highly conserved olfactory receptor among insects ${ }^{39}$ (over 550-850 million years old ${ }^{40}$ ). Curiously, attraction to $\mathrm{CO}_{2}$ in mosquitoes (as well as members of Coleoptera (Tribolium castaneum) and Lepidoptera (Bombyx morl)) is mediated—at least in part-by a system homologous to the Gr63a/Gr21a gustatory receptors that mediate aversion in Drosophila ${ }^{41}$. Other insect species that respond to $\mathrm{CO}_{2}$, including members of Hymenoptera (honeybees ${ }^{42}$ and ants ${ }^{12}$ ), Hemiptera (bed bugs ${ }^{3}$ and kissing bugs ${ }^{43}$ ), Blattodea (termites ${ }^{11}$ ), and Ixodida $\left(\right.$ ticks $\left.^{4}\right)$, however, lack this receptor ${ }^{41}$. It is possible that these insects also use the same evolutionarily ancient IR25a dependent $\mathrm{CO}_{2}$ pathway that is responsible for attraction in Drosophila.

The different time course in attraction to $\mathrm{CO}_{2}$ and ethanol, as well as the state-dependent decision to move towards or away from $\mathrm{CO}_{2}$, make this system ripe for exploring ecologically relevant decision making. Unfortunately, the GAL4 driver for the IR25a promoter is only expressed in about half of the endogenous IR25a-expressing neurons ${ }^{44}$, making imaging, silencing, and activation experiments difficult to interpret at this time. By narrowing the possible pathways of $\mathrm{CO}_{2}$ and ethanol attraction to IR25a, we hope to motivate future efforts to develop new genetic reagents that will make it possible to study this system in greater detail.

\section{Acknowledgements}

We to thank Andrew Straw for providing the 3D tracking software for our flight experiments. Richard Benton offered helpful feedback on an early draft of the manuscript and also provided the IR8a; IR25a; Orco, Gr63a quadruple mutant. Ralf Stanewsky provided an IR25a mutant and the IR25a + BAC rescue line. Greg Suh provided an IR8a mutant. Elizabeth Hong and Jeff Riffell contributed helpful comments on the manuscript. 


\section{References}

1. Dekker, T. \& Cardé, R. T. Moment-to-moment flight manoeuvres of the female yellow fever mosquito (Aedes aegypti L.) in response to plumes of carbon dioxide and human skin odour. J. Exp. Biol. 214, 3480-94 (2011).

2. van Breugel, F., Riffell, J., Fairhall, A. \& Dickinson, M. H. Mosquitoes Use Vision to Associate Odor Plumes with Thermal Targets. Curr. Biol. 25, 2123-2129 (2015).

3. Wang, C., Gibb, T., Bennett, G. W. \& McKnight, S. Bed Bug (Heteroptera: Cimicidae) Attraction to Pitfall Traps Baited With Carbon Dioxide, Heat, and Chemical Lure. J. Econ. Entomol. 102, 1580-1585 (2009).

4. Falco, R. C. \& Fish, D. Horizontal Movement of Adult Ixodes dammini (Acari: Ixodidae) Attracted to Co2-Baited Traps. J. Med. Entomol. 28, 726-729 (1991).

5. Guerenstein, P. G., Lorenzo, M. G., Núñez, J. A. \& Lazzari, C. R. Baker's yeast, an attractant for baiting traps for Chagas' disease vectors. Experientia 51, 834-837 (1995).

6. Anderson, J. R. \& Hoy, J. B. Relationship Between Host Attack Rates and Co2-Baited Insect Flight Trap Catches of Certain Symphoromyia Species. J. Med. Entomol. 9, 373-393 (1972).

7. Thom, C., Guerenstein, P. G., Mechaber, W. L. \& Hildebrand, J. G. Floral CO2 Reveals Flower Profitability to Moths. J. Chem. Ecol. 30, 1285-1288 (2004).

8. Galbreath, R. A. Orientation of grass grub Costelytra zealandica (Coleoptera:Scarabaeidae) to a carbon dioxide source. New Zeal. Entomol. 11, 6-7 (1988).

9. Bernklau, E. J. \& Bjostad, L. B. Reinvestigation of Host Location by Western Corn Rootworm Larvae (Coleoptera: Chrysomelidae): CO2 Is the Only Volatile Attractant. J. Econ. Entomol. 91, 1331-1340 (1998).

10. Kojima, W. Attraction to Carbon Dioxide from Feeding Resources and Conspecific Neighbours in Larvae of the Rhinoceros Beetle Trypoxylus dichotomus. PLoS One 1-11 (2015). doi:10.5061/dryad.52473

11. Bernklau, E. J., Fromm, E. A., Judd, T. M. \& Bjostad, L. B. Attraction of Subterranean Termites (Isoptera) to Carbon Dioxide. J. Econ. Entomol. 98, 476-484 (2005).

12. Buehlmann, C., Hansson, B. S. \& Knaden, M. Path Integration Controls Nest-Plume Following in Desert Ants. Curr. Biol. 22, 645-649 (2012).

13. Stange, G. Carbon Dioxide Is a Close-Range Oviposition Attractant in the Queensland Fruit Fly Bactrocera tryoni. Naturwissenschaften 86, 190-192 (1999).

14. Klocke, D., Schmitz, A. \& Schmitz, H. Native flies attracted to bushfires. (2009).

15. Suh, G. S. B. et al. A single population of olfactory sensory neurons mediates an innate avoidance behaviour in Drosophila. Nature 431, 854-859 (2004).

16. Faucher, C., Forstreuter, M., Hilker, M. \& de Bruyne, M. Behavioral responses of Drosophila to biogenic levels of carbon dioxide depend on life-stage, sex and olfactory context. J. Exp. Biol. 209, 2739-2748 (2006).

17. Suh, G. S. B., Leon, S. B. De, Benzer, S., Tanimoto, H. \& Anderson, D. J. Light Activation of an Innate Olfactory Avoidance Response in Drosophila. Curr. Biol. 2, 905-908 (2007).

18. Jones, W. D., Cayirlioglu, P., Grunwald Kadow, I. \& Vosshall, L. B. Two chemosensory receptors together mediate carbon dioxide detection in Drosophila. Nature 445, 86-90 (2007).

19.
Turner, S. L. \& Ray, A. Modification of CO2 avoidance behavior in Drosophila by inhibitory odorants. Nature 461, 277-282 (2009).

20. Ai, M. et al. Acid sensing by the Drosophila olfactory system. Nature 468, 691-695 (2010).

21. Faucher, P., Hilker, M. \& de Bruyne, M. Interactions of Carbon Dioxide and Food Odours in Drosophila : Olfactory Hedonics and Sensory Neuron Properties. PLoS One 8, 1-13 (2013).

22. Lin, H., Chu, L., Fu, T., Dickson, B. J. \& Chiang, A. Parallel Neural Pathways Mediate CO 2 Avoidance Responses in Drosophila. Science (80-. ). 340, 1338-1341 (2013).

23. Su, C., Menuz, K., Reisert, J. \& Carlson, J. R. Non-synaptic inhibition between grouped neurons in an olfactory circuit. Nature 492, 66-71 (2012).

24. Sachse, S. et al. Activity-Dependent Plasticity in an Olfactory Circuit. Neuron 56, 838-850 (2007).

25. Bell, J. S. \& Wilson, R. I. Behavior Reveals Selective Summation and Max Pooling among Olfactory Processing Channels. Neuron 91, 425-438 (2016).

26. Michael L. Sorey et al. Invisible CO2 Gas Killing Trees at Mammoth Mountain, California. (2001).

27. Hubbard, H. G. Insect Life in the Hot Springs of Yellowstone National Park. Can. Entomol. 23, (1891).

28. Straw, A. D., Branson, K., Neumann, T. R. \& Dickinson, M. H. Multi-camera real-time three-dimensional tracking of multiple flying animals. J. R. Soc. Interface 8, 395-409 (2011).

29. van Breugel, F. \& Dickinson, M. H. Plume-tracking behavior of flying Drosophila emerges from a set of distinct sensorymotor reflexes. Curr. Biol. 24, 274-86 (2014).

30. Wasserman, S., Salomon, A. \& Frye, M. a. Drosophila tracks carbon dioxide in flight. Curr. Biol. 23, 301-6 (2013).

31. Yorozu, S. et al. Distinct sensory representations of wind and near-field sound in the Drosophila brain. Nature 457, 201-205 (2009).

32. Gaudry, Q., Nagel, K. I. \& Wilson, R. I. Smelling on the fly: sensory cues and strategies for olfactory navigation in Drosophila. Curr. Opin. Neurobiol. 22, 216-22 (2012).

33. Kwon, J. Y., Dahanukar, A., Weiss, L. a \& Carlson, J. R. The molecular basis of CO2 reception in Drosophila. Proc. Natl. Acad. Sci. U. S. A. 104, 3574-8 (2007).

34. Chen, C. et al. Drosophila Ionotropic Receptor 25a mediates circadian clock resetting by temperature. Nature $\mathbf{5 2 7}, 516-520$ (2015).

35. Lighton, J. R. B. Discontinuous Gas Exchange in Insects. Annu. Rev. Entomol. 41, 309-324 (1996).

36. Hetz, S. K. \& Bradley, T. J. Insects breathe discontinuously to avoid oxygen toxicity. Nature 433, 516-519 (2005).

37. M. Dicke, J C van Lenteren, G J F Boskamp, E. van D. L. Chemical stimuli in host-habitat location by Leptopilina heterotoma (Thomson) (Hymenoptera: Eucoilidae), a parasite of Drosophila. J. Chem. Ecol. 10, 695-712 (1984).

38. Guerenstein, P. G. \& Hildebrand, J. G. Roles and Effects of Environmental Carbon Dioxide in Insect Life. Annu. Rev. Entomol. 161-178 (2008).

doi:10.1146/annurev.ento.53.103106.093402

39. Silbering, A. F. et al. Complementary Function and Integrated Wiring of the Evolutionarily Distinct Drosophila Olfactory Subsystems. J. Neurosici. 31, 13357-13375 (2011).

40. Croset, V. et al. Ancient protostome origin of chemosensory ionotropic glutamate receptors and the evolution of insect taste and olfaction. PLoS Genet. 6, 1-20 (2010). 
41. Robertson, H. M. \& Kent, L. B. Evolution of the gene lineage encoding the carbon dioxide receptor in insects. J. insect Sci. 9, 19 (2009).

42. Seeley, T. D. Atmospheric carbon dioxide regulation honeybee (apis mellifera) colonies. J. Insect Physiol. 20, 2301-2305 (1974).

43. Barrozo, R. B., Minoli, S. A. \& Lazzari, C. R. Circadian rhythm of behavioural responsiveness to carbon dioxide in the bloodsucking bug Triatoma infestans (Heteroptera: Reduviidae ). J. Insect Physiol. 50, 249-254 (2004).

44. Ulbrich, M. H., Isacoff, E. Y., Kellenberger, S., Abuin, L. \& Benton, R. Functional Architecture of Olfactory Ionotropic Glutamate Receptors. Neuron 69, 44-60 (2011). 